\title{
Activities, Procedures and Doses in Pediatric Patients Due to Radiopharmaceuticals
}

\author{
Silvia Maria Velasques de Oliveira ${ }^{*}$, Edson Mendes Boasquevisque ${ }^{2}$, Felipe Sima dos \\ Santos ${ }^{1}$ and Rodrigo Biancardi ${ }^{1}$ \\ ${ }^{1}$ Instituto de Radioproteção e Dosimetria, Av. Salvador Allende, s/n; 22780166; silvia@ird.gov.br; Rio de Janeiro - \\ RJ - Brasil. ${ }^{2}$ Faculdade de Ciências Médicas; Universidade do Estado do Rio de Janeiro; Av. 28 de Setembro, 87; \\ 20551030; Rio de Janeiro - RJ - Brasil
}

\begin{abstract}
An investigation performed between 2003 and 2005 in sixteen selected public and private institutions in Northeast, Southeast and South geographical regions of Brazil evaluated average organ doses and effective doses in 2,411 pediatric patients due to diagnostic procedures with radiopharmaceuticals. For 1 year, effective doses were greater than literature. For 5 years, differences were noticed between present work and literature for bone scintigraphy, thyroid scintigraphy and ${ }^{67} \mathrm{Ga}$ citrate scintigraphy. These differences may be attributed to the uncertainties in internal dose calculations. High absorved doses in bone surfaces of children due to ${ }^{67}$ Ga citrate and bone scintigraphy should be evaluated accordingly. Current protocols used recommend standardized mean activities per mean weight for all ages. However, it was observed that the activities were not standardized and were higher for children with younger ages. Future studies are needed for optimising activities of radiopharmaceuticals to these patients in the country.
\end{abstract}

Keywords: pediatrics, oncology, dosimetry, radiopharmaceuticals, nuclear medicine, radiation protection

\section{INTRODUCTION}

Currently, about $70 \%$ of children and adolescents with cancer may be cured if diagnosis is precocious. In Brazil, the incidence of pediatric cancer is about $3 \%$ of the total. For 2008, they are expected about ten thousand new cases of cancer in children and adolescents behind 18 years (Inca, 2008). In general, male are the majority of malignant tumours in childhood. The radiation protection desires special consideration. Considering the prognosis of cure, it must be reduced the probability of stochastic effects of radiation which may appear in the mature years. Effective doses are related to long-term effects of ionising radiation. They are a weighted sum of the equivalent doses received by each organ and the applied weighting factors depend on the sensitivity of each organ. In nuclear medicine, the effective dose mainly depends on the applied radionuclide, the anatomy of the patient, the biokinetics of the tracer and the administered activity (Jacobs, 2005). An investigation performed (2003-2005) in sixteen selected public and private institutions in Northeast, Southeast and South regions of Brazil, which are responsible for $92 \%$ of

\footnotetext{
* Author for correspondence
} 
radiopharmaceuticals consumption and nuclear medicine imaging equipments (Velasques de liveira, 2005). This article presents the investigation results.

\section{MATERIAL AND METHODS}

A questionnaire was send to the institutions, requesting: number of procedures per year, number of patients per gender and age, individual weight and height, and activities of radiopharmaceuticals per procedure. In four institutions, the researcher himself collected data. For determining the real activity administered, strings were measured into the ionisation chamber two times: immediately before and after injection.

It was observed if mean corporal weight ranged per age was similar to simulators used by ICRP. Thus, the absorved doses to organs and effective doses were obtained by multiplying mean activity per procedure per dose conversion factors, as appropriate (ICRP 1987, 1998).

It was used $\mathrm{T}$ test for homogeneity of independent samples and Pearson correlation for "activity" as independent variable and the others as dependent. It was used SPSS for Windows v.10.0 linear regression "stepwise" ( $\mathrm{F} \leq 0.050$, excluding all values $\mathrm{p} \geq 0.10$ ).

\section{RESULTS AND DISCUSSION}

The most frequent diagnostic procedures were: bone scintigraphy (37\%); renal studies (18\%); $67 \mathrm{Ga}$ citrate scintigraphy (12\%); myocardial (9\%) and lung (9\%) perfusion studies; whole body scanning (7\%); liver and spleen $(2 \%)$ and thyroid studies (2\%); brain perfusion (2\%) and testes imaging $(1 \%)$. Other procedures contributed with less than $2 \%$.

There were studied 2,411 children protocols, which 1,286 were male (53\%), 992 female (41\%) and $133(6 \%)$ had no identification. Per age, 208 patients had between 0 and 1 year (8\%), 305 had more than 1 until 2 years (13\%), 607 had more than 2 until 7 years (25\%), 669 had more than 7 until 12 years $(28 \%)$ and 466 had more than 12 until 17 years (18\%). Age was not registered in 156 children (6\%). Individual weight was registered in 257 patients (less than 11\%) and mean corporal weight for all age intervals was compatible with Cristy mathematical phantoms (ICRP, 1975) (Table 1).

Table 1 - Comparisons between children corporal weight $(\mathrm{kg})$ and Cristy phantoms (ICRP, 1975) and fractions of adult activities used for estimating activities to be used for children $(\mathrm{n}=257)$.

\begin{tabular}{cccccc}
\hline \multirow{2}{*}{$\begin{array}{c}\text { Age range } \\
\text { (years) }\end{array}$} & $\begin{array}{c}\text { Patients } \\
(\mathbf{n})\end{array}$ & \multicolumn{2}{c}{ Corporal weight $(\mathbf{k g})$} & \multicolumn{2}{c}{ Fraction of adult activities used for children } \\
\cline { 3 - 6 } & & Present work & $\begin{array}{c}\text { Cristy } \\
\text { phantoms }\end{array}$ & ARSAC (2000) & Present work \\
\hline $0-0,9$ & 28 & $5,0-13,4$ & - & $0,2-0,3$ & $0,2-0,3$ \\
$1-2$ & 46 & $8,0-22,0$ & 10 & $0,2-0,5$ & $0,3-0,4$ \\
$>2-7$ & 74 & $13,5-24,0$ & 18 & $0,4-0,5$ & 0,4 \\
$>7-12$ & 69 & $26,5-55,7$ & 33 & $0,5-0,9$ & $0,5-0,7$ \\
$>12-17$ & 40 & $29,3-75,0$ & 55 & $0,6-0,9$ & $0,6-0,9$ \\
$0-17$ & 257 & $5,0-75,0$ & $10-55$ & $0,2-0,9$ & $0,2-0,9$ \\
\hline
\end{tabular}

It was noticed a great dispersion in patients corporal weight between 12 and 17 years (29.3$75.0 \mathrm{~kg}$ ). The criteria "fraction of the adult activities used for children" (ARSAC, 2000) were followed (Table 1).There was no correlation between "activities" and "age" and "weight" and strong correlation between "age" and "weight" $(\mathrm{r}=0.812 ; \mathrm{p}<0.05)$. Current protocols recommend standardized mean activities per mean weight for all ages (SNM, 1997; Hahn et al., 2001; Guianmarile et al, 2008). However, in the present study, mean activities per mean weight were not standardized and were higher for children with younger ages (Figure 1). 


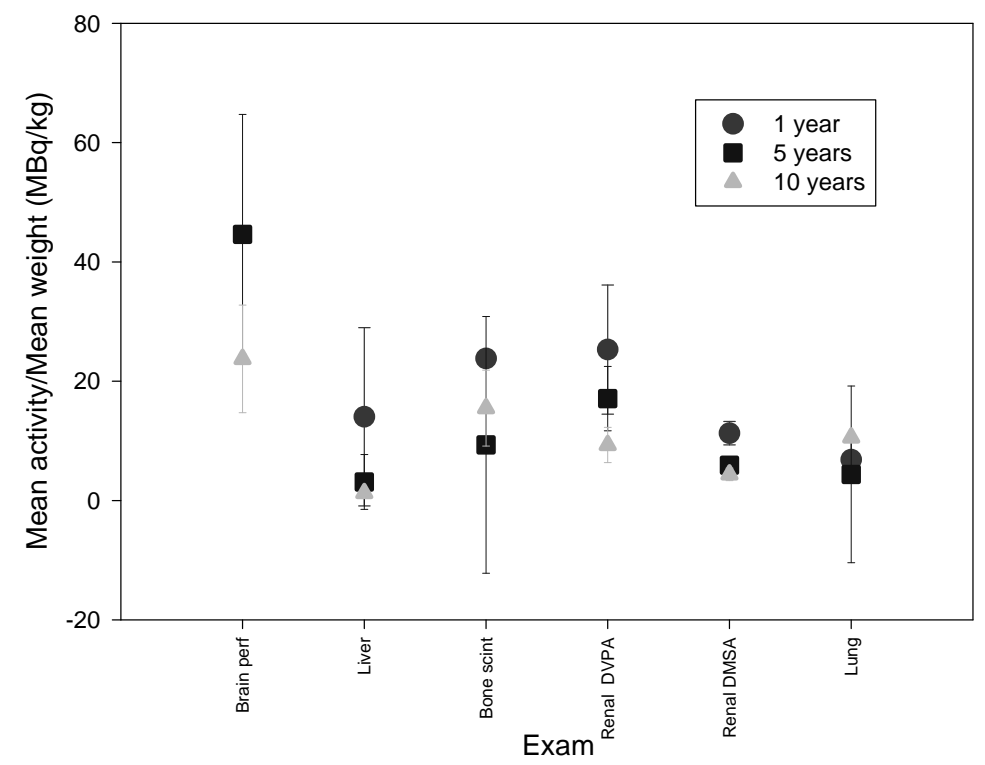

Figure 1 - Mean activities per mean weight $(\mathrm{MBq} / \mathrm{kg})$ for children per exam. The uncertainty is expressed by confidence interval of $95 \%(n=162)$.

For 1 year, effective doses were greater than literature (Stabin and Gelfand, 1998) (Table 2). For 5 years, differences were noticed between present work and literature (Stabin and Gelfand, 1998) for bone scintigraphy with $99 \mathrm{mTc}$ MDP, respectively $(7 \pm 2) \mathrm{mSv}$ and $2,7 \mathrm{mSv}$, thyroid scintigraphy with $99 \mathrm{mTc}$ pertechnetate, respectively $(5 \pm 2) \mathrm{mSv}$ and $1.2 \mathrm{mSv}$ and $67 \mathrm{Ga}$ citrate, respectively $(29 \pm 1) \mathrm{mSv}$ and $21 \mathrm{mSv}$
(Table 2). Other procedures had effective doses below $5 \mathrm{mSv}$. For myocardial perfusion with 99mTc Sestamibi, effective doses were lower than literature (Stabin and Gelfand, 1998) for 5 years (Table 2). Bone surfaces absorved doses due to 67Ga citrate imaging and bone scintigraphy with 99mTc MDP were, respectively, $(386 \pm 108) \mathrm{mGy}$ and $(197 \pm 103) \mathrm{mGy}$ for 1 year and $(195 \pm 24) \mathrm{mGy}$ and $(112 \pm 27) \mathrm{mGy}$ for 5 years (Table 3$)$.

Table 2 - Mean effective doses (mSv) for children of 1 and 5 years in present work and literature (Stabin and Gelfand, 1998) ( $\mathrm{p}<0.05)$.

\begin{tabular}{|c|c|c|c|c|}
\hline \multirow[b]{2}{*}{ Procedures/Radiopharmaceuticals } & \multicolumn{2}{|c|}{1 year $(\mathrm{mSv})$} & \multicolumn{2}{|c|}{5 years $(\mathrm{mSv})$} \\
\hline & $\begin{array}{c}\text { Present } \\
\text { work }\end{array}$ & $\begin{array}{c}\text { Stabin and } \\
\text { Gelfand (1998) }\end{array}$ & $\begin{array}{c}\text { Present } \\
\text { work }\end{array}$ & $\begin{array}{c}\text { Stabin and Gelfand } \\
(\mathbf{1 9 9 8 )}\end{array}$ \\
\hline Bone scintigraphy ${ }^{99 \mathrm{~m}} \mathrm{Tc}$ MDP & $10 \pm 5$ & 2.8 & $7 \pm 2$ & 2.7 \\
\hline Renal imaging ${ }^{99 \mathrm{~m}} \mathrm{Tc}$ DMSA & $3 \pm 2$ & 0.7 & $2 \pm 1$ & 0.8 \\
\hline Renal imaging ${ }^{99 \mathrm{~m}} \mathrm{Tc}$ DTPA & $3 \pm 1$ & 0.3 & $2 \pm 1$ & 0.4 \\
\hline Renogram ${ }^{99 \mathrm{~m}}$ Tc MAG-3 & $2 \pm 0.7$ & 0.3 & $1.3 \pm 0.5$ & 0.6 \\
\hline $\begin{array}{l}\text { Liver and spleen imaging with }{ }^{99 \mathrm{~m}} \mathrm{Tc} \\
\text { sulphur colloid }\end{array}$ & $3 \pm 1$ & 0.8 & $2 \pm 0.6$ & 0.8 \\
\hline Lung perfusion ${ }^{99 \mathrm{~m}} \mathrm{Tc}$ MAA & $4 \pm 1$ & 1.3 & $3 \pm 0.2$ & 1.2 \\
\hline Myocardial perfusion ${ }^{99 \mathrm{~m}} \mathrm{Tc}$ Sestamibi & $6^{\ddagger}$ & $5^{¥}$ & 3.1 & 5.7 \\
\hline Thyroid image ${ }^{99 \mathrm{~m}} \mathrm{Tc}$ pertechnetate & - & - & $5 \pm 2$ & 1.2 \\
\hline${ }^{67}$ Ga citrate scintigraphy & $48 \pm 13$ & 18 & $29 \pm 1$ & 21 \\
\hline
\end{tabular}

\footnotetext{
${ }^{¥}$ Activities were standardized per age.
} 
Table 3 - Mean activities administered (MBq), mean absorved organ doses (mGy) and mean effective doses (mSv) per procedures ranged per age (years) $(n=1,336)$.

\begin{tabular}{|c|c|c|c|c|c|c|c|}
\hline \multirow{2}{*}{$\begin{array}{c}\text { Procedure/ } \\
\text { Radiopharmaceutical }\end{array}$} & \multirow[b]{2}{*}{$\mathbf{n}$} & \multirow{2}{*}{$\begin{array}{l}\text { Mean activity } \\
\quad(\mathrm{MBq})\end{array}$} & \multicolumn{4}{|c|}{ Absorved organ doses (mGy) } & \multirow{2}{*}{$\begin{array}{l}\text { Effective } \\
\text { dose }(m S v)\end{array}$} \\
\hline & & & Bone surfaces & $\begin{array}{c}\text { Red } \\
\text { marrow }\end{array}$ & Kidneys & Bladder & \\
\hline \multicolumn{8}{|c|}{1 year $(n=228)$} \\
\hline $\begin{array}{l}\text { Bone scintigraphy }{ }^{99 \mathrm{~m}} \mathrm{Tc} \\
\text { MDP }\end{array}$ & 122 & $366 \pm 197$ & $197 \pm 103$ & $25 \pm 13$ & $12 \pm 6$ & $48 \pm 25$ & $10 \pm 5$ \\
\hline $\begin{array}{l}\text { Renal scintigraphy }{ }^{99 \mathrm{~m}} \mathrm{Tc} \\
\text { DMSA }\end{array}$ & 52 & $88 \pm 43$ & $5 \pm 3$ & $1.2 \pm 1$ & $67 \pm 33$ & $5 \pm 3$ & $3 \pm 2$ \\
\hline${ }^{67} \mathrm{Ga}$ citrate scintigraphy & 36 & $105 \pm 94$ & $386 \pm 108$ & $111 \pm 30$ & $54 \pm 48$ & $39 \pm 35$ & $48 \pm 13$ \\
\hline${ }^{131} \mathrm{I}-\mathrm{MIBG}$ & 18 & $52 \pm 5$ & $19 \pm 6$ & $18 \pm 6$ & $27 \pm 9$ & $118 \pm 57$ & - \\
\hline \multicolumn{8}{|c|}{5 years $(n=399)$} \\
\hline $\begin{array}{l}\text { Bone scintigraphy }{ }^{99 \mathrm{~m}} \mathrm{Tc} \\
\text { MDP }\end{array}$ & 248 & $510 \pm 122$ & $112 \pm 27$ & $17 \pm 4$ & $9 \pm 2$ & $37.3 \pm 9.0$ & $7 \pm 2$ \\
\hline $\begin{array}{l}\text { Renal scintigraphy } \\
{ }^{99 \mathrm{~m}} \mathrm{Tc} \text { DMSA }\end{array}$ & 74 & $107 \pm 30$ & $2 \pm 0.4$ & $1.0 \pm 0.3$ & $46 \pm 12$ & $3 \pm 1$ & $2 \pm 0.6$ \\
\hline${ }^{67} \mathrm{Ga}$ citrate scintigraphy & 58 & $140 \pm 145$ & $195 \pm 24$ & $63 \pm 8$ & $40.5 \pm 42.0$ & $28.0 \pm 29.0$ & $29 \pm 1.2$ \\
\hline${ }^{131} \mathrm{I}-\mathrm{MIBG}$ & 19 & $69 \pm 21$ & $12 \pm 4$ & $13 \pm 4$ & $21 \pm 6$ & $116.5 \pm 35$ & - \\
\hline \multicolumn{8}{|c|}{10 years $(n=448)$} \\
\hline $\begin{array}{l}\text { Bone scintigraphy }{ }^{99 \mathrm{~m}} \mathrm{Tc} \\
\text { MDP }\end{array}$ & 317 & $546 \pm 127$ & $72 \pm 15$ & $9 \pm 2$ & $6.6 \pm 1.5$ & $48.4 \pm 10.3$ & $6 \pm 1$ \\
\hline $\begin{array}{l}\text { Renal scintigraphy } \\
{ }^{99 \mathrm{~m}} \text { Tc DMSA }\end{array}$ & 48 & $124 \pm 40$ & $1 \pm 0.4$ & $1 \pm 0.3$ & $37 \pm 12$ & $4 \pm 1$ & $2 \pm 0.6$ \\
\hline${ }^{67}$ Ga citrate scintigraphy & 69 & $150 \pm 141$ & $142 \pm 8$ & $41.6 \pm 2.4$ & $30 \pm 28$ & $23 \pm 21$ & $22 \pm 1$ \\
\hline${ }^{131} \mathrm{I}-\mathrm{MIBG}$ & 14 & $78 \pm 25$ & $9 \pm 3$ & $10 \pm 3$ & $16 \pm 5$ & $57 \pm 19$ & - \\
\hline \multicolumn{8}{|c|}{15 years $(n=261)$} \\
\hline $\begin{array}{l}\text { Bone scintigraphy }{ }^{99 \mathrm{~m}} \mathrm{Tc} \\
\text { MDP }\end{array}$ & 175 & $642 \pm 162$ & $54 \pm 11$ & $7 \pm 1$ & $6 \pm 1$ & $39 \pm 8$ & $5 \pm 1$ \\
\hline $\begin{array}{l}\text { Renal scintigraphy } \\
{ }^{99 \mathrm{~m}} \text { Tc DMSA }\end{array}$ & 31 & $198 \pm 48$ & $1.2 \pm 0.3$ & $1 \pm 0.2$ & $44 \pm 10.5$ & $5 \pm 1$ & $2 \pm 0.5$ \\
\hline${ }^{67}$ Ga citrate scintigraphy & 55 & $116 \pm 16$ & $94 \pm 13$ & $27 \pm 4$ & $16 \pm 2$ & $13 \pm 2$ & $15 \pm 2$ \\
\hline
\end{tabular}


For 131I-MIBG pre-treatment screening, the absorved doses in bladder wall were $(118 \pm 57)$ $\mathrm{mGy}$ and $(117 \pm 35) \mathrm{mGy}$ for 1 year and 5 years, respectively (Table 3), but higher doses were absorved in liver and spleen: (241 \pm 80$) \mathrm{mGy}$, $(164 \pm 49) \mathrm{mGy}$ and $(124 \pm 41) \mathrm{mGy}$ for liver and (168 \pm 56$) \mathrm{mGy},(117 \pm 35) \mathrm{mGy}$ and $(86 \pm 28) \mathrm{mGy}$ for spleen, respectively for 1 year, 5 years and 10 years.

It must be emphasized that, depending on specific protocols, several $67 \mathrm{Ga}$ citrate or 131I-MIBG whole-body studies and bone scintigraphy may be requested. Nevertheless, the estimated mean doses do not may be applied to a single patient, but to a group of patients with similar characteristics.

\section{CONCLUSIONS}

The combined uncertainties (phantom and biokinetic parameters and variations in the tissueweighting factors over time), in any given radiopharmaceutical dose estimate are typically, at a minimum, a factor of 2 and may be considerably greater, in general, because of normal human variability, and particularly in disease states (Stabin, 2008). The differences observed in the effective doses between the present work and the literature may be attributed to these uncertainties.

Recent studies (Jacobs, 2005) recommend new methodology to determine activities per procedure for children and adolescents (Holm, 2007). Future research may allow optimising protocols for pediatric patients. It shall be evaluated if the estimated absorved doses to bone surfaces due to ${ }^{99 \mathrm{~m}} \mathrm{Tc}$ MDP and ${ }^{67} \mathrm{Ga}$ represent additional radiological risk to the patients, considering their special clinical conditions.

\section{ACKNOWLEDGEMENTS}

The authors thank to the staffs of collaborating institution and to Dr. L. Tahuata for technical assistance.

\section{RESUMO}

Foi realizado no Brasil, no período 2003-2005, um estudo sobre doses absorvidas em órgãos e doses efetivas devido ao uso de radiofármacos em pacientes pediátricos. Foram estudadas 2.411 crianças e adolescentes menores de 18 anos. Foi observado que as atividades usadas não foram padronizadas, sendo maiores para crianças de menor idade, podendo ser otimizadas conforme apropriado. Para 1 ano, as doses efetivas foram maiores do que as publicadas na literatura e para 5 anos, foram observadas diferenças para cintilografias ósseas, cintilografias da tireóide, e pesquisas de corpo inteiro com citrato de ${ }^{67} \mathrm{G}$. Deve ser avaliado se doses absorvidas em órgãos, especialmente para superfície óssea devido a cintilografias ósseas com ${ }^{99 \mathrm{~m}} \mathrm{Tc}$ MDP e pesquisa de corpo inteiro com citrato de ${ }^{67} \mathrm{Ga}$ podem acarretar risco radiológico adicional aos pacientes, considerando-se as peculiaridades de seu estado clínico.

\section{REFERENCES}

ARSAC (Administered Radioactive Substances Advisory Committee) (2000), Notes for guidance on the clinical administration of radiopharmaceuticals and use of sealed sources administered. Nuc Med Comm., 21.

Guianmarile, F.; Chiti, A.; Lassmann, M.; Boudewijn, B.; Flux, F. (2008), EANM procedures guidelines for ${ }^{131}$ I-metaiodobenzylguanidine $\left({ }^{131} \mathrm{I}-\mathrm{mIBG}\right)$ therapy. Eur J Nucl Med Mol Imaging, 35, 1039-1047.

Hahn, K.; Fisher, S.; Colarinha, P.; Gordon, I.; Mann M.; Piepz, A.; Olivier, P. (2001), Guidelines for bone scintigraphy in children. Eur J Nucl Med Mol Imaging, 28, 3.

Holm S., Borgwardt L., Loft A., Graff J., Law I.,Hojgaard L (2007). Pediatric doses-a critical appraisal of the EANM pediatric dosage card. Eur $J$ Nucl Med Mol Imaging, 34, 1713-1718.

Instituto Nacional do Câncer (INCA). Incidência de Câncer no Brasil (In Portuguese). www.inca.gov.br/estimativa2008/index/asp (accessed in 06.23.2008).

International Commission for Radiological Protection (1975), Report to the Task Group Reference Man, Publication 23, Annals of ICRP, Oxford Pergamon Press.

International Commission for Radiological Protection (1987), Radiation dose to patients from radiopharmaceuticals. Publication 53, Annals of ICRP, 18(1-4), Oxford Pergamon Press.

International Commission for Radiological Protection (1998), Radiation dose to patients from radiopharmaceuticals. Publication 80 , addendum to ICRP Publication 53, Annals of ICRP, 22 (3), Oxford Pergamon Press. 
Jacobs F., Thierens H., Piepsz A., Bacher K., Van de Wiele C., Ham H., Dierckx R.A. (2005). Optimised tracer-dependent dosage cards to obtain weightindependent effective doses. Eur J Nucl Med Mol Imaging, 32, 581-588.

Piepsz, A.; Hahn, K.; Roca, I.; Roca, I.; Ciofetta, G.; Toth, G.; Gordon, I.; Kolinska, J.; Gwidlet, J. (1990), A radiopharmaceuticals schedule for imaging in pediatrics. Eur J Nuc Med., 17, 127-129.

Society of Nuclear Medicine (SNM) (1997), Guidelines of Society of Nuclear Medicine, USA.

Stabin, M. G.; Gelfand, G. (1998), Dosimetry of pediatric nuclear medicine procedures. Quartely $J$ Nucl Med., 42, 93-112.
Stabin, M.G. (2008). Uncertainties in internal dose calculations for radiopharmaceuticals. J Nucl Med., 49, 853-860.

Velasques de Oliveira, S. M. (2005), Procedures, activities and doses in the cycle of nuclear medicine in Brazil, PhD Thesis (In Portuguese), Rio de Janeiro State University, Rio de Janeiro.

Received: August 20, 2008; Revised: September 03, 2008; Accepted: September 06, 2008. 